\title{
Sur la quantité de nombres économiques
}

\author{
par \\ Jean-Marie De Koninck (Québec), Nicolas Doyon (Québec) \\ et Florian LuCA (Morelia)
}

1. Introduction. En 1995, Bernardo Recamán Santos [7] a défini un nombre économique comme étant un entier positif dont la factorisation ne requiert pas plus de chiffres que sa représentation décimale. Ainsi, $25=5^{2}$ est un nombre économique en base 10. De manière analogue, on a introduit la notion de nombre économique en base $B \geq 2$. Par ailleurs, on dit qu'un nombre est fortement économique en base $B$ si sa factorisation nécessite moins de chiffres que son écriture en base $B$. Par exemple, $125=5^{3}$ est un nombre fortement économique en base 10 .

Répondant à une question posée par Santos [7], De Koninck et Luca [4] ont démontré qu'il existe des suites arbitrairement longues de nombres fortement économiques consécutifs, un résultat déjà obtenu par Pinch [5] mais sous la condition de la conjecture des nombres premiers jumeaux généralisée.

Récemment, De Koninck et Luca [3] ont obtenu des bornes inférieure et supérieure pour la fonction $N_{B}(x)$, soit la quantité de nombres fortement économiques n'excédant pas $x$. Nous améliorons ces bornes en obtenant une formule asymptotique pour la quantité $\log x-\log N_{B}(x)$.

2. Le résultat principal. Dans tout ce qui suit, $B$ est un entier $\geq 2$, $x$ est un grand nombre réel et $p$ désigne toujours un nombre premier. Pour chaque entier $n \geq 2$, on désigne respectivement par $\omega(n)$ et $\Omega(n)$ le nombre de facteurs premiers distincts de $n$ et le nombre de facteurs premiers de $n$ en comptant leur multiplicité. Enfin $\{x\}$ désigne la partie fractionnaire du nombre réel $x$.

Nous utilisons de plus les symboles $\gg$ et $\ll$ de Vinogradov ainsi que les symboles $O$ et $o$ de Landau avec leurs significations habituelles.

2000 Mathematics Subject Classification: 11A63, 11A25. 
Introduisons la fonction $R$ définie pour tout nombre réel $\alpha$ par

$$
R(\alpha)= \begin{cases}0 & \text { si } \alpha \leq 0 \\ \min _{t>0} \frac{1-e^{-t}}{t} e^{\alpha t} & \text { si } 0<\alpha<1 / 2, \\ 1 & \text { si } \alpha \geq 1 / 2\end{cases}
$$

Il est clair que cette fonction est bien définie. Par ailleurs, soit $t_{0}(\alpha)$ la valeur de $t$ qui minimise l'expression $\left(1-e^{-t}\right) e^{\alpha t} / t$. On constate alors que

$$
t_{0}(\alpha)=(1+o(1)) \frac{1}{\alpha} \quad(\alpha \rightarrow 0) .
$$

Il s'ensuit que $R(\alpha)$ est continue en $\alpha=0$. D'autre part, comme il est clair que $R(\alpha)$ est continue en $\alpha=1 / 2$, on peut conclure que $R$ est une fonction continue sur l'ensemble des nombres réels. La figure 1 illustre la fonction $R(\alpha)$.

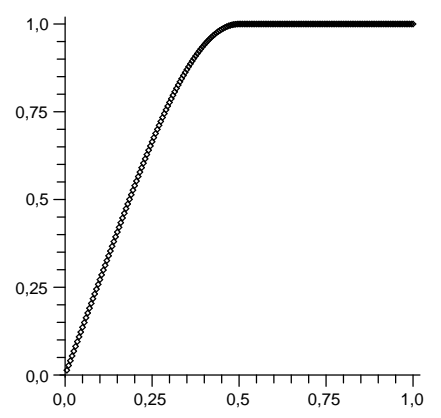

Fig. 1. La fonction $R(\alpha)$

Notre résultat principal consiste en des bornes inférieure et supérieure pour la fonction $N_{B}(x)$.

THÉORÈmE. Lorsque $x$ tend vers l'infini,

$$
N_{B}(x)=\frac{x}{(\log x)^{K(B)+o(1)}},
$$

où

$$
K(B)=\min _{\varrho>0}\left(\min _{0<\beta<1}(1+\varrho+\beta(\log \beta-1-\log (R(\varrho / \beta \log B))))\right) .
$$

En particulier, lorsque $B$ tend vers l'infini,

$$
K(B)=1-\frac{1+o(1)}{\log B} .
$$

Voici d'ailleurs un tableau donnant quelques valeurs numériques de la fonction $K(B)$. 


\begin{tabular}{ccccc}
\hline$B$ & $K(B)$ & & $B$ & $K(B)$ \\
\cline { 1 - 2 } \cline { 5 - 6 } 2 & 0,330 & & 8 & 0,696 \\
3 & 0,474 & & 9 & 0,713 \\
4 & 0,553 & & 10 & 0,727 \\
5 & 0,603 & & 20 & 0,789 \\
6 & 0,643 & & 100 & 0,868 \\
7 & 0,669 & & 1000 & 0,909 \\
\hline
\end{tabular}

3. Les résultats préliminaires. On désigne par $d_{B}(n)$ le nombre de chiffres nécessaires à l'écriture de la factorisation de $n$ en base $B$. On pose également $\gamma(1)=f_{B}(1)=1$ et pour $n \geq 2$,

$$
\gamma(n):=\prod_{p \mid n} p \quad \text { et } \quad f_{B}(n):=\sum_{p \mid n}\left(1-\left\{\frac{\log p}{\log B}\right\}\right) .
$$

Avec ces notations, on obtient l'identité

$$
\begin{aligned}
d_{B}(n) & =\sum_{p \mid n}\left\lfloor\frac{\log p}{\log B}+1\right\rfloor+\sum_{\substack{p^{a} \| n \\
a \geq 2}}\left\lfloor\frac{\log a}{\log B}+1\right\rfloor \\
& =\frac{\log \gamma(n)}{\log B}+f_{B}(n)+\sum_{\substack{p^{a} \| n \\
a \geq 2}}\left\lfloor\frac{\log a}{\log B}+1\right\rfloor .
\end{aligned}
$$

À l'aide de cette identité, on peut démontrer les deux lemmes suivants qui sont des critères sur les nombres économiques utilisés par De Koninck et Luca [3].

LEMME 1. Écrivons chaque entier $n \geq 2$ sous la forme $n=2^{a} r$, où $r$ est un nombre impair. La condition suivante est suffisante pour que $n$ soit un nombre économique:

$$
f_{B}(r)<\frac{a \log 2}{\log B}-4-\frac{\log \log n-\log \log 2}{\log B} .
$$

Démonstration. Il est clair que

$$
\begin{aligned}
d_{B}(n) & \leq 2+\left\lfloor\frac{\log a}{\log B}+1\right\rfloor+d_{B}(r) \leq 3+\frac{\log a}{\log B}+\left\lfloor\frac{\log r}{\log B}+1\right\rfloor+f_{B}(r) \\
& \leq 4+\frac{\log a}{\log B}+\frac{\log r}{\log B}+f_{B}(r) .
\end{aligned}
$$

Il s'ensuit que $n$ est un nombre économique si

$$
4+\frac{\log a}{\log B}+\frac{\log r}{\log B}+f_{B}(r) \leq \frac{\log n}{\log B}=\frac{\log r+a \log 2}{\log B} .
$$


Il en découle que $n$ est un nombre économique si

$$
f_{B}(r) \leq \frac{a \log 2}{\log B}-4-\frac{\log a}{\log B}
$$

et le lemme 1 découle alors du fait que $a \leq \log n / \log 2$.

On pose

$$
v=v(n):=\prod_{\substack{p^{a} \| n \\ a>1}} p^{a}
$$

et $m=m(n):=n / v$, de sorte que $v(n)$ est la partie puissante de $n$ et $m(n)$ sa partie libre de carrés. On a alors le résultat suivant.

Lemme 2. Pour tout $\varepsilon>0$, il existe un entier positif $n_{0}$ tel que, pour tout $n>n_{0}$ avec $v(n)<(\log \log n)^{2}$, le nombre $n$ est fortement économique seulement si

$$
f_{B}(m)<\frac{1}{\log B}(\log v-\log \gamma(v))+\varepsilon(\log \log \log n)^{2} .
$$

Démonstration. De l'identité (3) on obtient

$$
d_{B}(n)=\frac{\log \gamma(n)}{\log B}+f_{B}(n)+\sum_{\substack{p^{a} \| n \\ a>1}}\left(\left\lfloor\frac{\log a}{\log B}\right\rfloor+1\right) .
$$

Observons d'une part que $\log \gamma(n)=\log n-\log v+\log \gamma(v)$ et d'autre part que

$f_{B}(n)=f_{B}(m)+f_{B}(v)=f_{B}(m)+O\left(\frac{\log v}{\log \log v}\right)=f_{B}(m)+o(\log \log \log n)$.

Pour établir cette dernière identité, on a utilisé l'hypothèse $v(n)<(\log \log n)^{2}$. Par ailleurs, toujours grâce à cette hypothèse,

$$
\sum_{\substack{p^{a} \| n \\ a>1}}\left\lfloor\frac{\log a}{\log B}+1\right\rfloor=O(\omega(v) \log v)=o\left((\log \log \log n)^{2}\right) .
$$

Il s'ensuit que

$$
d_{B}(n)=\frac{1}{\log B}(\log n-\log v+\log \gamma(v))+f_{B}(m)+o\left((\log \log \log n)^{2}\right),
$$

ce qui complète la démonstration du lemme 2 .

Introduisons maintenant la notion d'indice de composition d'un entier positif $n$, lequel est désigné par la fonction $\lambda(n)$ définie par $\lambda(1):=1$ et pour $n \geq 2$ par

$$
\lambda(n):=\frac{\log n}{\log \gamma(n)},
$$

une fonction d'abord introduite par Jerzy Browkin dans [1]. 
Posons maintenant, pour chaque entier $j \geq 0$ et chaque nombre réel $\delta>0$

$$
H_{j, \delta}(t):=\#\{n \leq t: 2+j \delta \leq \lambda(n) \leq 2+(j+1) \delta\} .
$$

Lemme 3. Pour $j$ et $\delta$ fixés,

$$
H_{j, \delta}(t)=t^{1 /(2+j \delta)+o(1)} \quad(t \rightarrow \infty) .
$$

Démonstration. Voir De Koninck et Doyon [2].

Soit $G=G(k)=g_{1}+\cdots+g_{k}$ la somme de $k$ variables aléatoires indépendantes chacune uniformément distribuée sur $[0,1]$.

Lemme 4. Soit $\alpha$ un nombre réel fixe. Pour tout entier positif $k$,

$$
P(G<\alpha k) \leq R(\alpha)^{k} .
$$

De plus, pour tout $\varepsilon>0$, il existe une constante $C=C(\varepsilon, \alpha)>0$ et un entier positif $k_{0}$ tels que pour tout $k \geq k_{0}$,

$$
P(G<\alpha k) \geq \frac{1}{2} R(\alpha)^{k+(C+\varepsilon) k^{3 / 4}} .
$$

Démonstration. D'abord, dans le cas $\alpha \leq 0$, on a trivialement

$$
P(G<\alpha k)=P(G<0)=0=R(\alpha)^{k},
$$

ce qui établit d'un seul coup les deux inégalités du lemme.

Soit donc $\alpha>0$. Par symétrie, $P(G<\alpha k)=P(G>(1-\alpha) k)$, de sorte que $P(G<k / 2)=P(G>k / 2)=1 / 2$. Si $\alpha \geq 1 / 2$, alors $1 / 2 \leq P(G<\alpha k) \leq 1$, de sorte que par la définition de $R$, on a $P(G<\alpha k) \leq 1=R(\alpha)^{k}$, ce qui établit (6). D'autre part, il est clair que $\frac{1}{2} R(\alpha)^{k}=1 / 2 \leq P(G<\alpha k)$, ce qui établit (7) dans le cas où $\alpha \geq 1 / 2$.

Pour le reste de la preuve, nous supposerons donc que $0<\alpha<1 / 2$.

Pour une variable aléatoire $X$, désignons par $f_{X}$ la fonction de densité associée à $X$, et, étant donnée une fonction réelle $h$, désignons par $E[h(X)]$ l'espérance mathématique de $h(X)$.

Montrons d'abord (6). En utilisant le fait que les variables aléatoires $g_{i}$, pour $i=1, \ldots, k$, sont indépendantes et identiquement distribuées, pour tout nombre réel $t$ on a

$$
\int_{0}^{k} e^{-t x} f_{G}(x) d x=E\left[e^{-t G}\right]=\left(E\left[e^{-t g_{1}}\right]\right)^{k}=\left(\frac{1-e^{-t}}{t}\right)^{k} .
$$

Cela permet d'écrire que pour tout $t>0$,

$$
P(G<\alpha k)=\int_{0}^{\alpha k} f_{G}(x) d x<e^{t \alpha k} \int_{0}^{\alpha k} e^{-t x} f_{G}(x) d x<\left(\frac{e^{\alpha t}\left(1-e^{-t}\right)}{t}\right)^{k} .
$$


Il s'ensuit que

$$
P(G<\alpha k) \leq\left(\min _{t>0}\left(\frac{e^{t \alpha}\left(1-e^{-t}\right)}{t}\right)\right)^{k}=R(\alpha)^{k}
$$

ce qui prouve (6) lorsque $0<\alpha<1 / 2$.

Pour établir (7), nous allons d'abord borner la fonction de densité $f_{G}(x)$. De l'inégalité (9), il découle que pour tout $0<\varepsilon<\alpha$,

$$
\int_{(\alpha-\varepsilon) k}^{\alpha k} f_{G}(x) d x=P((\alpha-\varepsilon) k \leq G<\alpha k) \leq P(G<\alpha k) \leq R(\alpha)^{k} .
$$

Par ailleurs, comme $f_{G}(x)$ est une fonction continue et croissante sur $[0, k / 2]$, il s'ensuit que

$$
\int_{(\alpha-\varepsilon) k}^{\alpha k} f_{G}(x) d x \geq \varepsilon k f_{G}((\alpha-\varepsilon) k)
$$

En combinant (10) et (11), on en déduit que

$$
\varepsilon k f_{G}((\alpha-\varepsilon) k) \leq R(\alpha)^{k}
$$

laquelle inégalité, en posant $\alpha-\varepsilon=\alpha^{\prime}$, devient

$$
f_{G}\left(\alpha^{\prime} k\right) \leq \frac{R\left(\alpha^{\prime}+\varepsilon\right)^{k}}{k \varepsilon}
$$

Comme la fonction $R$ est uniformément continue, il existe une constante $c>0$ telle que $R\left(\alpha^{\prime}+\varepsilon\right)<R\left(\alpha^{\prime}\right)+c \varepsilon$. En choisissant $\varepsilon=R\left(\alpha^{\prime}\right) / k$, il découle de (12) que

$$
f_{G}\left(\alpha^{\prime} k\right) \leq \frac{\left(R\left(\alpha^{\prime}\right)+c R\left(\alpha^{\prime}\right) / k\right)^{k}}{R\left(\alpha^{\prime}\right)} \leq R\left(\alpha^{\prime}\right)^{k-1} e^{c} .
$$

Pour simplifier les notations, posons $c_{1}:=e^{c}$ et remplaçons $\alpha^{\prime}$ par $\alpha$, de sorte que (13) devient

$$
f_{G}(\alpha k)<c_{1} R(\alpha)^{k-1}
$$

Les relations (8) et (14) nous permettent de conclure que, pour tout $\varepsilon>0$ et tout $t>0$, on a la chaîne d'inégalités

$$
\begin{aligned}
& \int_{(\alpha-\varepsilon) k}^{(\alpha+\varepsilon) k} f_{G}(x) e^{-t x} d x \\
& \quad=\left(\frac{1-e^{-t}}{t}\right)^{k}-\int_{0}^{(\alpha-\varepsilon) k} f_{G}(x) e^{-t x} d x-\int_{(\alpha+\varepsilon) k}^{k} f_{G}(x) e^{-t x} d x
\end{aligned}
$$




$$
\begin{aligned}
& \geq\left(\frac{1-e^{-t}}{t}\right)^{k}-k \max _{x \notin[(\alpha-\varepsilon) k,(\alpha+\varepsilon) k]} f_{G}(x) e^{-t x} \\
& \geq\left(\frac{1-e^{-t}}{t}\right)^{k}-k \max _{x \notin[(\alpha-\varepsilon) k,(\alpha+\varepsilon) k]} c_{1} R(x / k)^{k-1} e^{-t x} .
\end{aligned}
$$

Comme ces inégalités sont valides pour tout $t>0$, on peut choisir $t=t_{0}(\alpha)$. On obtient dans ce cas

$$
\max _{x \notin[(\alpha-\varepsilon) k,(\alpha+\varepsilon) k]} R(x / k)^{k-1} e^{-t_{0}(\alpha) x}=\max _{\theta \notin[\alpha-\varepsilon, \alpha+\varepsilon]}\left(e^{-t_{0}(\alpha) \theta} R(\theta)\right)^{k} R(\theta)^{-1} .
$$

Il découle de la définition de la fonction $R$ que

$$
e^{-t_{0}(\alpha) \theta} R(\theta)=e^{-t_{0}(\alpha) \theta} \frac{e^{t_{0}(\theta) \theta}\left(1-e^{-t_{0}(\theta)}\right)}{t_{0}(\theta)} .
$$

D'autre part, comme $R(\theta)$ est en réalité une fonction des variables $\theta$ et $t_{0}(\theta)$, on peut écrire $R(\theta)=S\left(\theta, t_{0}(\theta)\right)$, de sorte que

$$
\begin{aligned}
\frac{d}{d \theta} e^{-t_{0}(\alpha) \theta} R(\theta) & =-t_{0}(\alpha) e^{-t_{0}(\alpha) \theta} R(\theta)+e^{-t_{0}(\alpha) \theta} \frac{d R(\theta)}{d \theta} \\
& =-t_{0}(\alpha) e^{-t_{0}(\alpha) \theta} R(\theta)+e^{-t_{0}(\alpha) \theta}\left(\frac{\partial S}{\partial t_{0}} \cdot \frac{\partial t_{0}}{\partial \theta}+\frac{\partial S}{\partial \theta}\right) .
\end{aligned}
$$

Or comme $t_{0}(\theta)$ a été choisi de façon à minimiser $R(\theta)$, il est clair que $\partial S / \partial t_{0}=0$. Puisque $\partial S / \partial \theta=t_{0}(\theta) R(\theta)$, il s'ensuit que

$$
\frac{d}{d \theta} e^{-t_{0}(\alpha) \theta} R(\theta)=e^{-t_{0}(\alpha) \theta} R(\theta)\left(t_{0}(\theta)-t_{0}(\alpha)\right) .
$$

Comme $e^{-t_{0}(\alpha) \theta} R(\theta)>0$ et comme $t_{0}(\theta)$ est une fonction décroissante, il suit que la fonction $e^{-t_{0}(\alpha) \theta} R(\theta)$ atteint son maximum lorsque $\theta=\alpha$, et en fait qu'elle est croissante sur $(0, \alpha)$ et décroissante sur $(\alpha, 1 / 2)$. C'est pourquoi

$$
\max _{\theta \notin[\alpha-\varepsilon, \alpha+\varepsilon]} e^{-t_{0}(\alpha) \theta} R(\theta) \leq e^{-t_{0}(\alpha) \alpha} R(\alpha)-c_{2}(\alpha) \varepsilon^{2}
$$

pour une certaine constante $c_{2}(\alpha)$ indépendante de $\varepsilon$. En choisissant $\varepsilon=$ $k^{-1 / 4}$, on obtient alors

$$
\begin{aligned}
k \max _{x \notin[(\alpha-\varepsilon) k,(\alpha+\varepsilon) k]} R(x / k)^{k-1} e^{-t_{0}(\alpha) x} & =O\left(k\left(e^{-t_{0}(\alpha) \alpha} R(\alpha)-c_{2} k^{-1 / 2}\right)^{k}\right) \\
& =o\left(\left(\frac{1-e^{-t_{0}(\alpha)}}{t_{0}(\alpha)}\right)^{k}\right) .
\end{aligned}
$$

En utilisant cette inégalité dans (15), on déduit que pour tout $\delta>0$, il existe un entier positif $k_{0}$ tel que pour tout $k \geq k_{0}$,

$$
\int_{\left(\alpha-k^{-1 / 4}\right) k}^{\left(\alpha+k^{-1 / 4}\right) k} f_{G}(x) e^{-t_{0}(\alpha) x} d x>(1-\delta)\left(\frac{1-e^{-t_{0}(\alpha)}}{t_{0}(\alpha)}\right)^{k} .
$$


En observant par ailleurs que

$$
\begin{aligned}
\int_{\left(\alpha-k^{-1 / 4}\right) k}^{\left(\alpha+k^{-1 / 4}\right) k} f_{G}(x) e^{-t_{0}(\alpha) x} d x & \leq e^{-t_{0}(\alpha)\left(\alpha-k^{-1 / 4}\right) k} \int_{\left(\alpha-k^{-1 / 4}\right) k}^{\left(\alpha+k^{-1 / 4}\right) k} f_{G}(x) d x \\
& \leq e^{-t_{0}(\alpha)\left(\alpha-k^{-1 / 4}\right) k} P\left(G<\left(\alpha+k^{-1 / 4}\right) k\right),
\end{aligned}
$$

on peut déduire de (16) que

$$
e^{-t_{0}(\alpha)\left(\alpha-k^{-1 / 4}\right) k} P\left(G<\left(\alpha+k^{-1 / 4}\right) k\right) \geq(1-\delta)\left(\frac{1-e^{-t_{0}(\alpha)}}{t_{0}(\alpha)}\right)^{k},
$$

et ainsi que

$$
P\left(G<\left(\alpha+k^{-1 / 4}\right) k\right) \geq(1-\delta)\left(\frac{e^{\alpha t_{0}(\alpha)}\left(1-e^{-t_{0}(\alpha)}\right)}{t_{0}(\alpha)}\right)^{k} e^{-t_{0}(\alpha) k^{3 / 4}} .
$$

En posant $\alpha^{\prime}=\alpha+k^{-1 / 4}$ et comme la fonction $e^{\alpha t_{0}(\alpha)}\left(1-e^{-t_{0}(\alpha)}\right) / t_{0}(\alpha)$ est continue, nous avons

$$
e^{\alpha^{\prime} t\left(\alpha^{\prime}\right)}\left(1-e^{-t\left(\alpha^{\prime}\right)}\right) / t\left(\alpha^{\prime}\right)>e^{\alpha t_{0}(\alpha)}\left(1-e^{-t_{0}(\alpha)}\right) / t_{0}(\alpha)-c_{3} k^{-1 / 4},
$$

où $c_{3}=c_{3}(\alpha)$ est une constante positive qui ne dépend pas de $k$. Ainsi, en utilisant (17), on trouve qu'il existe une constante positive $C$ dépendante de $\alpha$ mais indépendante de $k$ telle que

$$
\begin{aligned}
P\left(G<\alpha^{\prime} k\right) & \geq(1-\delta)\left(\frac{e^{\alpha^{\prime} t\left(\alpha^{\prime}\right)}\left(1-e^{-t\left(\alpha^{\prime}\right)}\right)}{t\left(\alpha^{\prime}\right)}+c_{3} k^{-1 / 4}\right)^{k} e^{t_{0}\left(\alpha^{\prime}\right) k^{3 / 4}} \\
& \geq \frac{1}{2}\left(\frac{e^{\alpha^{\prime} t\left(\alpha^{\prime}\right)}\left(1-e^{-t\left(\alpha^{\prime}\right)}\right)}{t\left(\alpha^{\prime}\right)}\right)^{k+C k^{3 / 4}},
\end{aligned}
$$

ce qui complète la démonstration du lemme 4.

Étant donné un nombre réel positif $x$ et un entier positif $k$ fixés, on subdivise l'ensemble des nombres premiers $p \leq x$ en des sous-ensembles disjoints $\pi_{0}, \pi_{1}, \ldots, \pi_{k-1}$ définis comme suit :

$$
p \in \pi_{j} \Leftrightarrow \frac{j}{k}<1-\left\{\frac{\log p}{\log B}\right\} \leq \frac{j+1}{k} .
$$

Ainsi, pour $B=10, k=3$ et $x=30$, on obtient

$$
\pi_{0}=\{5,7\}, \quad \pi_{1}=\{3,23,29\}, \quad \pi_{2}=\{2,11,13,17,19\} .
$$

Au même titre, on introduit les fonctions de compte $\omega_{0}(n), \omega_{1}(n), \ldots, \omega_{k-1}(n)$ définies par

$$
\omega_{j}(n)=\sum_{\substack{p \mid n \\ p \in \pi_{j}}} 1
$$


LEMme 5. Lorsque $x \rightarrow \infty$,

$$
\#\{n<x: \omega(n)=r\}=(1+o(1)) D(z) \frac{x}{\log x} \frac{(\log \log x)^{r-1}}{(r-1) !},
$$

où $z=r / \log \log x$ et

$$
D(z):=\frac{1}{\Gamma(z+1)} \prod_{p}\left(1+\frac{z}{p-1}\right)\left(1-\frac{1}{p}\right)^{z} .
$$

Cette estimation est valable uniformément pour $x \geq 3$ et $1 \leq r \leq C \log \log x$ pour toute constante fixe $C>0$.

Démonstration. Il s'agit d'un résultat classique de Selberg [8].

Remarquons que sur $[0,1]$ la fonction $D(z)$ est croissante et que $D(1)=1$. Afin d'être en mesure d'utiliser le lemme 5, nous aurons également besoin du lemme technique suivant.

Lemme 6. Soient $x$ et $y$ deux nombres réels tels que $0<y \leq x$. Alors, lorsque $x \rightarrow \infty$,

$$
\#\{n<y: \omega(n)=\lfloor\beta \log \log x\rfloor\}=(\log x)^{o(1)} y(\log y)^{-\kappa \beta \log \kappa \beta+\kappa \beta-1},
$$

où $\kappa=\kappa(x, y):=\log \log x / \log \log y$.

Démonstration. En utilisant la formule de Stirling sous la forme $n$ ! = $(1+o(1)) \sqrt{2 \pi n}(n / e)^{n}$ ainsi que le résultat de Selberg (lemme 5), on obtient aisément

$$
\begin{aligned}
\#\{n<y: \omega(n)=\lfloor\beta & \log \log x\rfloor\} \\
& =(1+o(1)) D(\kappa \beta) \frac{y}{\log y} \frac{(\log \log y)^{\lfloor\beta \log \log x\rfloor-1}}{(\lfloor\beta \log \log x\rfloor-1) !} \\
& =(\log x)^{o(1)} \frac{y}{\log y}\left(\frac{e}{\kappa \beta}\right)^{\kappa \beta \log \log y} \\
& =(\log x)^{o(1)} y(\log y)^{-\kappa \beta \log \kappa \beta+\kappa \beta-1} .
\end{aligned}
$$

Signalons d'abord que, lorsque $y=x$, i.e. $\kappa=1$, le lemme 6 entraîne en particulier que

$$
\#\{n<x: \omega(n)=\lfloor\beta \log \log x\rfloor\}=x(\log x)^{-\beta \log \beta+\beta-1+o(1)},
$$

ce qui permet de déduire que

$$
\#\{n<x: \omega>10 \log \log x\}=o\left(\frac{x}{\log x}\right) .
$$


Lemme 7. Soit $\mathcal{A}$ un ensemble de nombres premiers tel que

$$
\sum_{\substack{p \in \mathcal{A} \\ p<x}} \frac{1}{p}=(1+o(1)) c \log \log x
$$

pour une certaine constante positive $c$. Alors

$\#\{n<x: p \mid n \Rightarrow p \in \mathcal{A}, \omega(n)=K\}=(1+o(1)) c^{K} \#\{n<x: \omega(n)=K\}$.

Démonstration. Il s'agit d'un cas particulier du théorème B de De Koninck et Luca [3].

Des lemmes 6 et 7 , on peut aisément déduire le corollaire suivant.

Corollaire 1. Soit $\mathcal{A}$ un ensemble de nombres premiers tel que

$$
\sum_{\substack{p \in \mathcal{A} \\ p<x}} \frac{1}{p}=(1+o(1)) c \log \log x
$$

pour une certaine constante positive $c$. Alors

$$
\begin{array}{r}
\#\{n<y: p \mid n \Rightarrow p \in \mathcal{A}, \omega(n)=\lfloor\beta \log \log x\rfloor\} \\
=(\log x)^{o(1)} y(\log y)^{-\kappa \beta \log \kappa \beta+\kappa \beta-1+\kappa \beta \log c .}
\end{array}
$$

Lemme 8. Pour tous nombres réels $a, b$ tels que $0 \leq a<b \leq 1$, on $a$

$$
\sum_{\substack{p<x \\ p / \log B\} \in[a, b]}} \frac{1}{p}=(1+o(1))(b-a) \log \log x .
$$

Démonstration. On a

$$
\sum_{\substack{p<x \\\{\log p / \log B\} \in[a, b]}} \frac{1}{p}=\sum_{0 \leq j<\log x / \log B} \sum_{B^{j+a}<p<B^{j+b}} \frac{1}{p} .
$$

D'après la formule de Mertens raffinée par Rosser et Schoenfeld [6], il existe une constante $c_{0}$ telle que

$$
\sum_{p<x} \frac{1}{p}=\log \log x+c_{0}+O\left(\frac{1}{\log ^{2} x}\right) .
$$

C'est pourquoi, on obtient

$$
\begin{aligned}
\sum_{B^{j+a}<p<B^{j+b}} \frac{1}{p} & =\log (j+b)-\log (j+a)+O\left(1 / j^{2}\right) \\
& =\log (1+b / j)-\log (1+a / j)+O\left(1 / j^{2}\right),
\end{aligned}
$$


de sorte que

$$
\begin{aligned}
\sum_{0 \leq j<\log x / \log B} & \sum_{B^{j+a}<p<B^{j+b}} \frac{1}{p} \\
= & \sum_{0 \leq j<\log x / \log B}(\log (1+b / j)-\log (1+a / j))+O(1) \\
= & \sum_{0 \leq j<\log x / \log B} \frac{b-a}{j}+O(1)=(1+o(1))(b-a) \log \log x
\end{aligned}
$$

ce qui complète la démonstration du lemme 8.

LEMme 9. Soit $k$ un entier positif fixe et $c_{0}, c_{1}, \ldots, c_{k-1}$ des nombres réels non négatifs fixes. Soient $x, y$ des nombres réels tels que $0<y \leq x$ et $\kappa:=\log \log x / \log \log y$. Alors, lorsque $x \rightarrow \infty$,

$$
\begin{aligned}
\#\left\{n \leq y: \omega_{i}(n)\right. & \left.=\left\lfloor c_{i} \log \log x\right\rfloor \text { pour } i=0,1, \ldots, k-1\right\} \\
& =(\log x)^{o(1)} y(\log y)^{-\kappa C_{k-1} \log k-\kappa D_{k-1}+C_{k-1}(\kappa-\log \kappa)-1},
\end{aligned}
$$

où $C_{j}:=c_{0}+c_{1}+\cdots+c_{j}$ et $D_{j}=c_{0} \log c_{0}+c_{1} \log c_{1}+\cdots+c_{j} \log c_{j}$ pour $j=0,1, \ldots, k-1$.

Remarque. Nous utiliserons le lemme 9 seulement dans le cas $y=x$, ce qui implique $\kappa=1$. Le membre de droite de la relation de l'énoncé se simplifie alors pour donner

$$
x(\log x)^{-C_{k-1} \log k-D_{k-1}+C_{k-1}-1+o(1)} .
$$

Par exemple, si $k=3$ et si $c_{0}=c_{1}=1 / 4$ et $c_{2}=1 / 2$, on obtient

$$
\begin{array}{r}
\#\left\{n<x: \omega_{0}(n)=\left\lfloor\frac{1}{4} \log \log x\right\rfloor, \omega_{1}(n)=\left\lfloor\frac{1}{4} \log \log x\right\rfloor, \omega_{2}(n)=\left\lfloor\frac{1}{2} \log \log x\right\rfloor\right\} \\
=x(\log x)^{-\log 3+\frac{3}{2} \log 2+o(1)} .
\end{array}
$$

Démonstration du lemme 9. Fixons $k \geq 1$. Soit $r$ un entier, $1 \leq r \leq k-1$. Supposons que le résultat soit vrai si $c_{j}=0$ pour tout $j \geq r$, et procédons par induction sur $r$. Pour $r=1$, le résultat découle immédiatement du corollaire 1. En supposant que le résultat soit vrai pour $r$, nous allons démontrer qu'il est alors vrai pour $r+1$.

Pour ce faire, introduisons d'abord les deux fonctions de compte

$$
\begin{aligned}
M(y):=\#\left\{n \leq y: \omega_{0}(n)=\omega_{1}(n)=\ldots=\omega_{r-1}(n)=0,\right. \\
\left.\omega_{r}(n)=\left\lfloor c_{r} \log \log x\right\rfloor, \omega_{r+1}(n)=\ldots=\omega_{k-1}(n)=0\right\}
\end{aligned}
$$

et

$$
T(y):=\#\{n \leq y: n \in T\}
$$


où

$$
\begin{aligned}
T:=\left\{n: \omega_{j}(n)=\left\lfloor c_{j} \log \log x\right\rfloor \operatorname{pour} j=0,1, \ldots, r-1\right. \\
\text { et } \left.\omega_{j}(n)=0 \text { pour } j \geq r\right\} .
\end{aligned}
$$

Remarquons que les fonctions $T$ et $M$ dépendent implicitement de $x$. On pose

$$
\nu:=\frac{\log \log (y / t)}{\log \log x}, \quad \mu:=\frac{\log \log t}{\log \log x} .
$$

Fort de ces notations, on a alors

$$
\begin{aligned}
& \#\left\{n \leq y: \omega_{j}(n)=\left\lfloor c_{j} \log \log x\right\rfloor \operatorname{pour} j=0,1, \ldots, r\right. \\
& \left.\quad \text { et } \omega_{j}(n)=0 \text { pour } j \geq r+1\right\} \\
& \quad=\sum_{t \in T} M\left(\frac{y}{t}\right)=\int_{1}^{y} M\left(\frac{y}{t}\right) d T(t) .
\end{aligned}
$$

En utilisant de nouveau le corollaire 1, pour chaque $t \leq y$ on obtient

$$
M\left(\frac{y}{t}\right)=(\log x)^{o(1)} \frac{y}{t}\left(\log \frac{y}{t}\right)^{-\nu c_{r} \log \nu c_{r}+\nu c_{r}-\nu c_{r} \log k-1} .
$$

Or, par notre hypothèse d'induction, on a

$$
T(t)=(\log x)^{o(1)} t(\log t)^{-\mu C_{r-1} \log \mu-\mu D_{r-1}+\mu C_{r-1}-\mu C_{r-1} \log k-1} .
$$

En utilisant $(20)$ et $(21)$, ainsi que les inégalités $\mu \leq \kappa, \nu \leq \kappa, \log t \leq \log y$ et $\log (y / t) \leq \log y$, on peut borner supérieurement l'intégrale apparaissant dans (19) et obtenir

$$
\begin{aligned}
\int_{1}^{x} M\left(\frac{x}{t}\right) d T(t) \leq & y(\log x)^{o(1)} \int_{1}^{y} \frac{1}{t}\left(\log \frac{y}{t}\right)^{-\kappa c_{r} \log \kappa+\kappa c_{r}-\kappa c_{r} \log k-1} \\
& \times(\log t)^{-\kappa C_{r-1} \log \kappa-\kappa D_{r-1}+\kappa C_{r-1}-\kappa C_{r-1} \log k-1} d t \\
= & (\log x)^{o(1)}(\log y)^{-\kappa C_{r} \log \kappa-\kappa D_{r}+\kappa C_{r}-\kappa C_{r} \log k-1} .
\end{aligned}
$$

En substituant (22) dans (19), la borne supérieure du lemme 9 suit immédiatement. Pour démontrer la borne inférieure, on utilise l'inégalité

$$
\int_{1}^{y} M\left(\frac{y}{t}\right) d T(t) \geq \int_{y^{1 / 3}}^{y^{2 / 3}} M\left(\frac{y}{t}\right) d T(t) .
$$

Dans cet intervalle, on a $\nu=\kappa+o(1)$ et $\mu=\kappa+o(1)$. On a également $\log t \geq \frac{1}{3} \log y$ et $\log (y / t) \geq \frac{1}{3} \log y$. Il s'ensuit que

$$
\begin{aligned}
\int_{1}^{y} M\left(\frac{y}{t}\right) d T(t) & \geq(\log x)^{o(1)} y\left(\frac{\log y}{9}\right)^{-\kappa C_{r} \log \kappa-\kappa D_{r}+\kappa C_{r}-\kappa C_{r} \log k-2} \int_{y^{1 / 3}}^{y^{2 / 3}} \frac{1}{t} d t \\
& =(\log x)^{o(1)}(\log y)^{-\kappa C_{r} \log \kappa-\kappa D_{r}+\kappa C_{r}-\kappa C_{r} \log k-1},
\end{aligned}
$$

ce qui termine la démonstration du lemme 9. 
Dans le cas $y=x$, i.e. $\kappa=1$, le lemme 9 peut également être écrit sous la forme suivante, ce qui nous permettra d'utiliser des résultats probabilistes.

Corollaire 2. Pour tout entier positif $k$ fixe et étant donné des entiers non négatifs $c_{0}, c_{1}, \ldots, c_{k-1}$, on a, lorsque $x \rightarrow \infty$,

$\#\left\{n \leq x: \omega_{j}(n)=\left\lfloor c_{j} \log \log x\right\rfloor\right.$ pour $\left.j=0,1, \ldots, k-1\right\}$

$$
\begin{aligned}
=\# & \left\{n \leq x: \omega(n)=\left\lfloor C_{k-1} \log \log x\right\rfloor\right\} \\
& \times k^{-\left\lfloor C_{k-1} \log \log x\right\rfloor} \frac{\left\lfloor C_{k-1} \log \log x\right\rfloor !}{\prod_{j=0}^{k-1}\left\lfloor c_{j} \log \log x\right\rfloor !}(\log x)^{o(1)} .
\end{aligned}
$$

Démonstration. Le résultat découle immédiatement des lemmes 5 et 9 et de la formule de Stirling.

De la définition de $f_{B}(n)$, il résulte immédiatement que

$$
\sum_{j=0}^{k-1} \frac{j \omega_{j}(n)}{k} \leq f_{B}(n) \leq \sum_{j=0}^{k-1} \frac{(j+1) \omega_{j}(n)}{k} .
$$

En posant également, pour $y \leq \log x$,

$$
F_{B}(x, y)=\#\left\{n \leq x: f_{B}(n)<y\right\}
$$

on déduit de (23) que

$$
F_{B}(x, y)<\sum_{r=1}^{\lfloor\log x\rfloor} \sum_{u \in S_{r}(y)} \#\left\{n<x: \omega_{0}(n)=u_{0}, \ldots, \omega_{k}(n)=u_{k}\right\},
$$

où $S_{r}(y)$ est l'ensemble des vecteurs $\left(u_{1}, \ldots, u_{k}\right)$ de dimension $k$ sur $\mathbb{N}$ tels que

$$
u_{1}+\cdots+u_{k}=r \quad \text { et } \quad \sum_{j=1}^{k} \frac{j u_{j}}{k}<y .
$$

En utilisant le corollaire 2 dans l'équation (24), on obtient

$$
F_{B}(x, y)<\sum_{r=1}^{\lfloor\log x / \log 2\rfloor} \#\{n<x: \omega(n)=r\} \frac{1}{k^{r}} \# S_{r}(y)(\log x)^{o(1)} .
$$

En désignant par $V_{r}$ la somme de $r$ variables indépendantes prenant chacune des valeurs $1 / k, 2 / k, \ldots, 1$ avec une probabilité de $1 / k$, on a alors l'identité

$$
\frac{1}{k^{r}} \# S_{r}(y)=P\left(V_{r}<y\right) \quad(r=1,2, \ldots) .
$$

C'est pourquoi il découle de (25) que

$$
F_{B}(x, y)<\sum_{r=1}^{\lfloor\log x / \log 2\rfloor} \#\{n<x: \omega(n)=r\} P\left(V_{r}<y\right)(\log x)^{o(1)} .
$$


De façon analogue, on peut montrer que

$$
F_{B}(x, y)>\sum_{r=1}^{\lfloor\log x / \log 2\rfloor} \#\{n<x: \omega(n)=r\} P\left(V_{r}<y-r / k\right) .
$$

Désignant maintenant par $G_{r}=g_{1}+\cdots+g_{r}$ la somme de $r$ variables indépendantes uniformément distribuées sur $[0,1]$, on obtient, pour tout $y$,

$$
P\left(V_{r}<y-r / k\right)<P\left(G_{r}<y\right)<P\left(V_{r}<y\right) .
$$

Comme $k$ peut être choisi arbitrairement grand, il s'ensuit que

$$
\lim _{k \rightarrow \infty} P\left(V_{r}<y-r / k\right)=P\left(V_{r}<y\right) .
$$

Ainsi, en combinant (26), (27) et (28), on en déduit que

$$
F_{B}(x, y)=\sum_{r=1}^{\lfloor\log x / \log 2\rfloor} \#\{n<x: \omega(n)=r\} P\left(G_{r}<y\right)(\log x)^{o(1)} .
$$

Corollaire 3 . Pour $2 \leq y \leq \log x$, lorsque $x \rightarrow \infty$,

$$
F_{B}(x, y)=\max _{r \leq 10 \log \log x} \#\{n<x: \omega(n)=r\} P\left(G_{r}<y\right)(\log x)^{o(1)}+o\left(\frac{x}{\log x}\right) .
$$

Démonstration. Le résultat découle aisément des relations (29) et (18), puisque l'on a alors

$$
\begin{aligned}
F_{B}(x, y)= & \sum_{r=1}^{\lfloor\log x\rfloor} \#\{n<x: \omega(n)=r\} P\left(G_{r}<y\right)(\log x)^{o(1)} \\
= & \max _{r \leq 10 \log \log x} \#\{n<x: \omega(n)=r\} P\left(G_{r}<y\right)(\log x)^{o(1)} \\
& +O(\#\{n \leq x: \omega(n)>10 \log \log x\}) \\
= & \max _{r \leq 10 \log \log x} \#\{n<x: \omega(n)=r\} P\left(G_{r}<y\right)(\log x)^{o(1)}+o\left(\frac{x}{\log x}\right) .
\end{aligned}
$$

Nous allons maintenant utiliser le corollaire 3 pour obtenir une estimation précise de l'expression $F_{B}(x, y)$ lorsque $y=\chi \log \log x$, où $\chi$ est un réel positif fixé et $x$ tend vers l'infini. D'abord, il est clair qu'il découle du corollaire 3 que

$$
\begin{aligned}
F_{B}(x, \chi \log \log x)= & (\log x)^{o(1)} \max _{1 \leq r \leq 10 \log \log x}(\#\{n<x: \omega(n)=r\} \\
& \left.\times P\left(G_{r}<\chi \log \log x\right)\right)+o\left(\frac{x}{\log x}\right),
\end{aligned}
$$

de sorte qu'en définissant le nombre $\beta=\beta(x, r)$ implicitement par l'équation 
$\lfloor\beta \log \log x\rfloor=r$, on obtient

$$
\begin{aligned}
& F_{B}(x, \chi \log \log x) \\
& =(\log x)^{o(1)} \max _{(\log \log x)^{-1} \leq \beta \leq 10}(\#\{n<x: \omega(n)=\lfloor\beta \log \log x\rfloor\} \\
& \left.\quad \times P\left(G_{\lfloor\beta \log \log x\rfloor}<\chi \log \log x\right)\right)+o\left(\frac{x}{\log x}\right) .
\end{aligned}
$$

En utilisant le lemme 6, on arrive alors à

$$
\text { (30) } \begin{aligned}
F_{B}(x, \chi \log \log x)= & (\log x)^{o(1)} \max _{(\log \log x)^{-1} \leq \beta \leq 10}\left(x(\log x)^{-\beta \log \beta+\beta-1}\right. \\
& \left.\times P\left(G_{\lfloor\beta \log \log x\rfloor}<\chi \log \log x\right)\right)+o\left(\frac{x}{\log x}\right) .
\end{aligned}
$$

Or, il découle du lemme 4 que

$$
\begin{aligned}
P\left(G_{\lfloor\beta \log \log x\rfloor}\right. & <\chi \log \log x) \\
= & (R(\chi / \beta)+o(1))^{\beta \log \log x}=(\log x)^{\beta \log R(\chi / \beta)+o(1)} .
\end{aligned}
$$

Des relations (30) et (31), on peut donc déduire que

$$
\begin{aligned}
& F_{B}(x, \chi \log \log x) \\
& =\max _{(\log \log x)^{-1} \leq \beta \leq 10}\left(x(\log x)^{\beta-1-\beta(\log \beta)+\beta \log R(\chi / \beta)+o(1)}\right),
\end{aligned}
$$

laquelle relation est fondamentale pour la démonstration du lemme qui suit.

LEMME 10. Lorsque $x \rightarrow \infty$,

$$
N_{B}(x)=\max _{0<\varrho<\log x / \log \log x} F_{B}\left(\frac{x}{(\log x)^{\varrho}}, \frac{\varrho}{\log B} \log \log x\right)(\log x)^{o(1)} .
$$

Démonstration. Nous allons d'abord démontrer que

$$
N_{B}(x) \geq \max _{0<\varrho<\log x / \log \log x} F_{B}\left(\frac{x}{(\log x) \varrho}, \frac{\varrho}{\log B} \log \log x\right) .
$$

Il découle du lemme 1 que

$$
N_{B}(x) \geq \max _{0 \leq a \leq \log x / \log 2} F_{B}\left(\frac{x}{2^{a}}, \frac{a \log 2}{\log B}\right) .
$$

Or, en définissant implicitement $\varrho$ par la relation $2^{a}=(\log x)^{\varrho}$, l'inégalité (33) suit immédiatement.

Nous allons maintenant établir que pour tout $\varepsilon>0$, il existe un nombre réel $x_{0}=x_{0}(\varepsilon)$ tel que pour tout $x>x_{0}$,

$$
N_{B}(x) \leq \max _{0<\varrho<\log x / \log \log x} F_{B}\left(\frac{x}{(\log x) \varrho}, \frac{\varrho}{\log B} \log \log x\right)(\log x)^{\varepsilon},
$$

laquelle inégalité combinée avec (33) complétera la preuve du lemme 10. 
Du lemme 2, on peut déduire que

$$
N_{B}(x) \leq \sum_{\substack{v<(\log x)^{2} \\ v \text { puissant }}} F_{B}\left(\frac{x}{v}, \frac{1}{\log B} \log \left(\frac{v}{\gamma(v)}\right)+\varepsilon \log \log x\right) .
$$

De la définition de la fonction $\lambda(n)$ donnée dans (5) on obtient

$$
\log \left(\frac{v}{\gamma(v)}\right)=\log v-\log \gamma(v)=\log v\left(1-\frac{1}{\lambda(v)}\right),
$$

ce qui nous permet d'écrire, en utilisant le fait que si $v$ est un nombre puissant alors $\lambda(v) \geq 2$,

$$
\begin{aligned}
N_{B}(x) & \leq \sum_{\substack{v<(\log x)^{2} \\
\lambda(v) \geq 2}} F_{B}\left(\frac{x}{v}, \frac{\log v}{\log B}\left(1-\frac{1}{\lambda(v)}\right)+\varepsilon \log \log x\right) \\
& =\sum_{\substack{v<(\log x)^{2} \\
2 \leq \lambda(v) \leq k_{1}}}+\sum_{\substack{v<(\log x)^{2} \\
\lambda(v)>k_{1}}}=T_{1}(x)+T_{2}(x),
\end{aligned}
$$

disons, où $k_{1}$ est un entier positif grand, mais fixe.

Pour évaluer $T_{2}(x)$, observons qu'il résulte du lemme 3 que

$$
\#\left\{v<(\log x)^{2}: \lambda(v) \geq k_{1}\right\}<(\log x)^{3 / k_{1}},
$$

de sorte que, en écrivant chaque nombre $v<(\log x)^{2}$ sous la forme $v=$ $(\log x)^{\xi}$, où $\xi \in(0,2)$, on peut déduire que

$$
\begin{aligned}
T_{2}(x) & <\sum_{\substack{v<(\log x)^{2} \\
\lambda(v) \geq k_{1}}} F_{B}\left(\frac{x}{v}, \frac{\log v}{\log B}+\varepsilon \log \log x\right) \\
& <(\log x)^{3 / k_{1}} \max _{0<\xi<2} F_{B}\left(\frac{x}{(\log x)^{\xi}}, \frac{\xi \log \log x}{\log B}+\varepsilon \log \log x\right) .
\end{aligned}
$$

Cherchons maintenant une borne supérieure pour $T_{1}(x)$, soit dans le cas où $\lambda(v)<k_{1}$. Fixons un petit nombre réel $\delta>0$. On peut alors écrire

$$
T_{1}(x)=\sum_{\substack{v<(\log x)^{2} \\ 2 \leq \lambda(v) \leq k_{1}}} F_{B}\left(\frac{x}{v}, \frac{\log v}{\log B}\left(1-\frac{1}{\lambda(v)}\right)+\varepsilon \log \log x\right)=\sum_{j=0}^{\left\lfloor k_{1} / \delta\right\rfloor} S_{j},
$$

où

$$
S_{j}:=\sum_{\substack{v<(\log x)^{2} \\ 2+j \delta \leq \lambda(v)<2+(j+1) \delta}} F_{B}\left(\frac{x}{v}, \frac{\log v}{\log B}\left(1-\frac{1}{\lambda(v)}\right)+\varepsilon \log \log x\right)
$$




$$
\begin{aligned}
< & \sum_{\substack{v<(\log x)^{2} \\
2+j \delta \leq \lambda(v)<2+(j+1) \delta}} F_{B}\left(\frac{x}{v}, \frac{\log v}{\log B}\left(1-\frac{1}{2+(j+1) \delta}\right)+\varepsilon \log \log x\right) \\
= & \int_{1}^{(\log x)^{2}} F_{B}\left(\frac{x}{t}, \frac{\log t}{\log B}\left(1-\frac{1}{2+(j+1) \delta}\right)(1+o(1))\right) d\left(H_{j, \delta}(t)\right)(\log x)^{o(1)} .
\end{aligned}
$$

En utilisant le lemme 3 pour évaluer cette dernière intégrale et en effectuant par la suite le changement de variable $t=(\log x)^{\xi}$, on peut remplacer (38) par

$$
\begin{aligned}
& S_{j} \leq \int_{1}^{(\log x)^{2}} F_{B}\left(\frac{x}{t}, \frac{\log t}{\log B}\left(1-\frac{1}{2+(j+1) \delta}\right)+\varepsilon \log \log x\right) \\
& \times t^{(2+j \delta)^{-1}-1+\varepsilon} d t \\
&= \int_{0}^{2}(\log x)^{\xi /(2+j \delta)} F_{B}\left(\frac{x}{(\log x)^{\xi}}, \frac{\xi \log \log x}{\log B}\left(1-\frac{1}{2+(j+1) \delta}\right)\right) \\
& \times(\log x)^{\varepsilon_{1}} d \xi
\end{aligned}
$$

où $\varepsilon_{1}=\varepsilon_{1}(\varepsilon)$ est un nombre réel positif qui tend vers 0 avec $\varepsilon$.

En faisant appel à la relation (32), nous obtenons une nouvelle borne supérieure pour $S_{j}$, soit

$$
\begin{aligned}
S_{j} & \leq \int_{0}^{2} F_{B}\left(\frac{x}{(\log x)^{\xi(1-1 /(2+j \delta))}}, \frac{\xi \log \log x}{\log B}\left(1-\frac{1}{2+(j+1) \delta}\right)\right) \\
& \leq 2 \max _{0 \leq \xi \leq 2} F_{B}\left(\frac{x}{(\log x)^{\xi}}, \frac{\xi}{\log B} \log \log x\right)^{\varepsilon_{2}} d \xi
\end{aligned}
$$

où $\varepsilon_{2}=\varepsilon_{2}\left(\varepsilon_{1}\right)$ est également une quantité positive qui tend vers 0 lorsque $\varepsilon_{1} \rightarrow 0$.

En substituant (40) dans (37), on obtient finalement

$$
\begin{aligned}
& \sum_{\substack{v<(\log x)^{2} \\
2 \leq \lambda(v) \leq k_{1}}} F_{B}\left(\frac{x}{v}, \frac{\log v}{\log B}\left(1-\frac{1}{2+(j+1) \delta}\right)+\varepsilon \log \log x\right) \\
& \quad \leq \frac{k_{1}}{\delta} \max _{0 \leq \xi \leq 2} F_{B}\left(\frac{x}{(\log x)^{\xi}}, \frac{\xi}{\log B} \log \log x\right)(\log x)^{\varepsilon_{2}} .
\end{aligned}
$$

En choisissant maintenant $k_{1}=3 / \varepsilon$ et $\delta=1 / k$, on peut alors déduire de (36) et (41) l'inégalité (35), complétant ainsi la preuve du lemme 10. 
4. La démonstration du résultat principal. D'abord, il est clair que la relation

$$
N_{B}(x)=\frac{x}{(\log x)^{K(B)+o(1)}}
$$

est une conséquence immédiate de l'équation (32) et du lemme 10.

Il reste à établir la relation (2). Pour ce faire, observons d'abord que le nombre $t=t_{0}(\alpha)$ auquel le minimum est atteint dans la définition de $R(\alpha)$ est donné par la solution de l'équation $\frac{d R}{d t}=0$, laquelle peut être écrite sous la forme

$$
\frac{-\left(1-e^{-t}\right) e^{t \alpha}}{t^{2}}+\frac{\alpha e^{\alpha t}+(1-\alpha) e^{-(1-\alpha) t}}{t}=0,
$$

ce qui se ramène après simplification à

$$
\frac{1}{t}-\frac{e^{-t}}{1-e^{-t}}=\alpha
$$

Il s'ensuit que lorsque $\alpha$ tend vers 0 , on a $t_{0}(\alpha) \sim 1 / \alpha$, de sorte que

$$
R(\alpha) \sim \alpha e .
$$

On voit donc que, lorsque $B$ tend vers l'infini,

$$
K(B)=(1+o(1)) \min _{\varrho>0}\left(\min _{0<\beta<1}(1+\varrho+\beta(\log \beta-1-\log (e \varrho / \beta \log B)))\right) .
$$

En dérivant l'expression à droite de (43) par rapport à $\beta$, on obtient

$$
2 \log \beta-\log (\alpha / \log B) \text {. }
$$

C'est pourquoi l'expression à droite de (43) est minimale lorsque

$$
\beta=\sqrt{\frac{\varrho}{\log B}} .
$$

En substituant cette valeur de $\beta$ dans l'expression à droite de (43), on obtient

$$
K(B)=(1+o(1)) \min _{\varrho>0}\left(1+\varrho-2 \sqrt{\frac{\varrho}{\log B}}\right) .
$$

Par ailleurs, en dérivant l'expression à droite de (44) par rapport à $\varrho$ et en l'égalant à 0 , on obtient

$$
1-\sqrt{\frac{1}{\varrho \log B}}=0
$$

de sorte que l'expression à droite de (44) est minimale lorsque

$$
\varrho=\frac{1}{\log B},
$$


auquel cas il découle de (44) que

$$
K(B)=(1+o(1))\left(1-\frac{1}{\log B}\right)
$$

ce qui prouve $(2)$ et complète la démonstration du théorème.

5. Remarque finale. La preuve du théorème ne dépend pas du fait que les nombres soient fortement économiques ou même tout simplement économiques. En fait, le théorème est valable pour toutes les fonctions

$$
N_{B}^{k}(x):=\#\left\{n \leq x:\left\lceil\frac{\log n}{\log B}\right\rceil<d_{B}(n)+k\right\},
$$

où $k$ est un entier positif satisfaisant à $k=o(\log \log x)$.

\section{Références}

[1] J. Browkin, The abc-conjecture, in: Number Theory, Trends Math., Birkhäuser, Basel, 2000, 75-105.

[2] J. M. De Koninck et N. Doyon, À propos de l'indice de composition des nombres, Monatsh. Math. 139 (2003), 151-167.

[3] J. M. De Koninck and F. Luca, Counting the number of economical numbers, Publ. Math. Debrecen 68 (2006), 97-133.

[4] - - - On strings of consecutive economical numbers of arbitrary length, Integers 5 (2005), no. 2, A5.

[5] R. Pinch, Economical numbers, http://www.chalcedon.demon.co.uk/publish.html\#62.

[6] J. B. Rosser and L. Schoenfeld, Sharper bounds for the Chebyshev functions $\theta(x)$ and $\psi(x)$, Math. Comp. 29 (1975), 243-269.

[7] B. R. Santos, Problem 2204. Equidigital representation, J. Recreational Math. 27 (1995), 58-59.

[8] A. Selberg, Note on a paper by L.G. Sathe, J. Indian Math. Soc. 18 (1954), 83-87.

Département de mathématiques et de statistique

Université Laval

Québec G1K 7P4, Canada

E-mail: jmdk@mat.ulaval.ca nicolas.doyon@mat.ulaval.ca
Mathematical Institute

UNAM

Ap. Postal 61-3 (Xangari)

CP 58089

Morelia, Michoacán, México E-mail: fluca@matmor.unam.mx 